Neurology India Official Publication of the Neurological Society of India July-August 2016 / Vol 64 / Issue 4
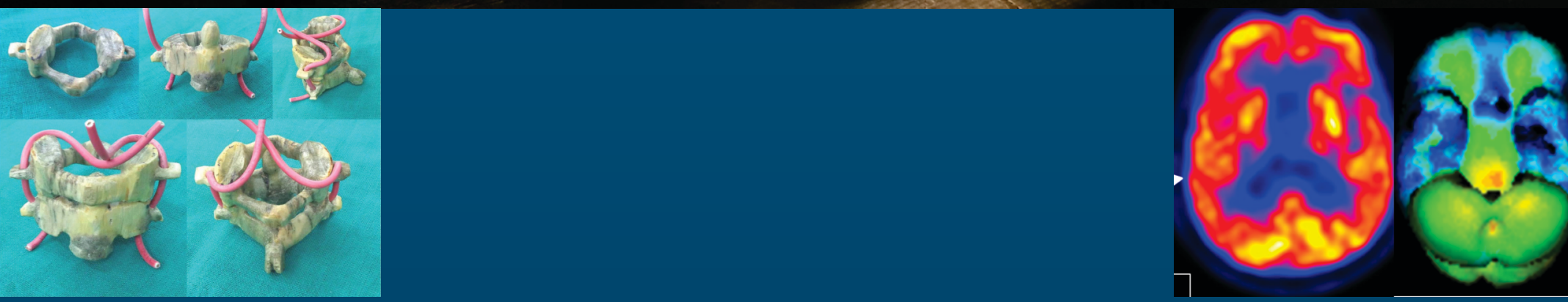

ISSN 0028-3886

www.neurologyindia.com 


\title{
Early neurological and cognitive impairments in subclinical cerebrovascular disease
}

\author{
Penka A. Atanassova, Radka I. Massaldjieva1, Borislav D. Dimitrov², Aleksandar S. Aleksandrov³, \\ Maria A. Semerdjieva ${ }^{1}$, Silvia B. Tsvetkova ${ }^{4}$, Nedka T. Chalakova, Kostadin A. Chompalov
}

Departments of Neurology and ${ }^{4}$ Radiology, Medical Faculty, Medical University, ${ }^{1}$ Department of Healthcare Management, Faculty of Public Health, Medical University, Plovdiv, ${ }^{3}$ Department of Cardiology, Military Medical Academy, Sofia, Bulgaria, ${ }^{2}$ Academic Unit of Primary Care and Population Sciences, Faculty of Medicine, University of Southampton, Southampton, United Kingdom

\begin{abstract}
Background: The subclinical cerebrovascular disease (SCVD) is an important public health problem with demonstrated prognostic significance for stroke, future cognitive decline, and progression to dementia. The earliest possible detection of the silent presence of SCVD in adults at age at risk with normal functioning is very important for both clinical doctors and scientists.

Materials and Methods: Seventy-seven adult volunteers, recruited during the years 2005-2007, with mean age 58.7 (standard deviation 5.9) years, were assessed by four subtests from the Cambridge Neuropsychological Test Automated Battery (CANTAB)-Eclipse cognitive assessment system. We used a questionnaire survey for the presence of cerebrovascular risk factors (CVRFs) such as arterial hypertension, smoking and dyslipidemia, among others, as well as instrumental (Doppler examination) and neurological magnetic resonance imaging (MRI) procedures. Descriptive statistics, comparison ( $t$-test, Chi-square) and univariate methods were used as followed by multifactor logistic regression and receiver operating characteristics analyses. Results: The risk factor questionnaire revealed nonspecific symptoms in 44 (67.7\%) of the subjects. In 42 (64.6\%) of all 65 subjects, we found at least one of the conventional CVRFs. Abnormal findings from the extra- and trans-cranial Doppler examination were established in 38 (58.5\%) of all studied volunteers. Thirty-four subjects had brain MRI (52.3\%), and abnormal findings were found in $12(35.3 \%)$ of them. Two of the four subtests of CANTAB tool appeared to be potentially promising predictors of the outcome, as found at the univariate analysis (spatial working memory 1 [SWM1] total errors; intra-extra dimensional set 1 [IED1] total errors [adjusted]; IED2 total trials [adjusted]). We established that the best accuracy of $82.5 \%$ was achieved by a multifactor interaction logistic regression model, with the role CVRF and combined CANTAB predictor "IED total ratio (errors/trials) $\times$ SWM1 total errors" $(P=0.006)$.

Conclusions: Our results have contributed to the hypothesis that it is possible to identify, by noninvasive methods, subjects at age at risk who have mild degree of cognitive impairment and to establish the significant relationship of this impairment with existing CVRFs, nonspecific symptoms and subclinical abnormal brain Doppler/MRI findings. We created a combined neuropsychological predictor that was able to clearly distinguish between the presence and absence of abnormal Doppler/ MRI findings. This pilot prognostic model showed a relatively high accuracy of $>80 \%$; therefore, the predictors may serve as biomarkers for SCVD in subjects at age at risk (51-65 years).
\end{abstract}

Key words: Cambridge Neuropsychological Test Automated Battery-Eclipse system; cerebrovascular risk factors; subclinical cerebrovascular disease

\begin{tabular}{|l|c|}
\hline \multicolumn{2}{|c|}{ Access this article online } \\
\hline \multirow{2}{*}{$\begin{array}{l}\text { Website: } \\
\text { www.neurologyindia.com }\end{array}$} & Quick Response Code \\
\hline DOI: & \\
\hline $10.4103 / 0028-3886.185359$ & \\
\hline & \\
& \\
\hline
\end{tabular}

Address for correspondence: Dr. Penka A. Atanassova, Department of Neurology, Medical Faculty, Medical University, 15A V. Aprilov Blvd., 4000 Plovdiv, Bulgaria.

E-mail: pp_atanassova@yahoo.com

This is an open access article distributed under the terms of the Creative Commons Attribution-NonCommercial-ShareAlike 3.0 License, which allows others to remix, tweak, and build upon the work non-commercially, as long as the author is credited and the new creations are licensed under the identical terms.

For reprints contact: reprints@medknow.com

How to cite this article: Atanassova PA, Massaldjieva RI, Dimitrov BD, Aleksandrov AS, Semerdjieva MA, Tsvetkova SB, et al. Early neurological and cognitive impairments in subclinical cerebrovascular disease. Neurol India 2016;64:646-55. 


\section{Introduction}

The "silent" cerebrovascular disease (CVD) is an important public health problem with demonstrated prognostic significance for stroke, future cognitive decline, and progression to dementia. If the type, combination, and prevalence of cerebrovascular risk factors (CVRFs) as well as the duration of their influence are sufficient to overcome the natural resistance of the cerebral circulation, the CVD begins. This stage has been recently called "subclinical CVD" (SCVD) by the Committee of the American Cardiological Association. ${ }^{[1]}$

Presence of subclinical disease in multiple vascular beds has been suggested as an indicator of the overall atherosclerotic burden. ${ }^{[2]}$ Consistent with this findings, investigators have reported an increased risk of overt CVD events in individuals with subclinical vascular disease. Such information may lead to further referencing in guidelines for the identification and treatment of individuals with higher probability of clinical cerebrovascular events. ${ }^{[3]}$

The earliest possible detection of the silent presence of SCVD in adults at age at risk, with normal functioning, is very important for both clinical doctors and scientists. We suppose that this preliminary stage of the disease progression is characterized by a mild degree of cognitive impairment (MDCI) that could be revealed and specified by relevant neuropsychological testing. Our work is based on the hypothesis that among adult individuals, MDCls with specific profile and severity could be found by neuropsychological screening tests and that these impairments may correlate with existing CVRFs and at the same time, with positive findings from brain Doppler examination and/or magnetic resonance imaging (MRI).

To test this hypothesis, we performed a cross-sectional study, named the Cambridge Neuropsychological Test Automated Battery (CANTAB)-SCVD project, with neurological, neuropsychological, Doppler, and MRI examinations of clinically healthy volunteers at age at risk (51-65 years) for "silent" presence of possible SCVD. Our aims were to (i) study the possible relationships between MDCls, CVRFs, and Doppler/MRI findings; (ii) identify a neuropsychological predictor, based on the finding from the neuropsychological assessment (CANTAB subtests) which may significantly discriminate between the presence and absence of positive findings from Doppler and/ or MRI; and (iii) create a diagnostic model for the risk of SCVD as characterized by these positive findings.

\section{Materials and Methods}

The CANTAB-SCVD project includes a population-based cross-sectional study of adult volunteers, designed to determine the feasibility of screening and detection of MDCls and positive findings by MRI and Doppler examinations.

\section{Patients' selection, diagnosis, and data collection}

Seventy-seven adult volunteers, recruited during the years 2005-2007 by neurologists from the Department of Neurology, Medical University Hospital, Plovdiv, satisfied all eligibility criteria. The inclusion criteria were (1) age between 51 and 65 years; and (2) availability of data on history for nonspecific complaints (yes/no) - headache, dizziness, tinnitus, slight changes in the attention and memory. The exclusion criteria were (1) previous stroke or transient ischemic attacks (TIAs), (2) previous myocardial infarction, (3) previous moderately severe or severe cranial traumatic injury, (4) psychiatric disorders, (5) epilepsy, (6) migraine, (7) peripheral vascular disease, and/or (8) neuroinfection sequel. All individuals included in this study provided written informed consent, according to the Declaration of Helsinki guidelines. The Ethics Committee of the Medical University, Plovdiv approved the study protocol.

We used a questionnaire survey for detecting the presence of the most frequent CVRFs (arterial hypertension, smoking, dyslipidemia, etc.) and for instrumental and neuroimaging procedures, the extra- and trans-cranial Doppler examination and the brain MRI.

The neuropsychological screening was performed with the CANTAB-Eclipse system, a language-independent test battery for cognitive impairments, with confirmed construct validity, ${ }^{[4,5]}$ using touch screen technology ${ }^{[4]}$ In this work, we present the results from 4 CANTAB subtests for impairments of working memory and strategy use, spatial planning and motor control, visual attention and attentional set shifting, and speed of response (Spatial Working Memory, SWM; Stockings of Cambridge, SOC; Rapid Visual Information Processing, RVP; and, Intra-Extra Dimensional Set, IED, respectively). The choice of these subtests was related to the expected impairment of attention and executive control in subjects with SCVD.

Several outcome measures for each CANTAB subtest were used in the statistical analyses. The level of performance of every subject was determined following the normative values as supplied by the CANTAB Eclipse PC Manager (personal communication).

\section{Statistical analyses}

Due to the pilot nature of this cross-sectional trial, a priori sample size was not calculated. Data are mean (standard deviation [SD] or standard error) or number and frequency (percentage), unless otherwise stated. The 
characteristics of the participants were assessed by methods of descriptive statistics, tests of normality and method of percentiles, and the two groups (with positive and negative Doppler/MRI findings) were compared by two-tailed independent sample Student's $t$-test, Mann-Whitney test, or chi-square test, as appropriate. All variables with complete datasets for each participant were included in the analyses. The variables with skewed distribution were normalized by log-transformation before the analyses.

The associations between different variables are listed in Table 1 and the findings from the Doppler/MRI examinations were evaluated by univariate analyses. Correlation analysis was performed by Pearson's $R$ coefficient or Spearman's $\rho$ coefficient, where appropriate. Logistic regression analysis was applied by entry and backward stepwise methods with adjustment for covariate effects (logit link function with likelihood ratio or conditional tests, as appropriate) to those variables that were significantly associated with the positive findings (outcome) at univariate analyses, without potential confounders. Some of the variables were used in combination to create combined predictors (e.g., combined neuropsychological predictor (CNPP) from CANTAB battery). Logistic curve estimation function was used to fit the regression models, with calculation of odds ratios (OR) and their $95 \%$ confidence intervals $(95 \% \mathrm{Cls})$. Two-tailed $P<0.05$ was considered statistically significant for all tests, correlations, and regressions. All analyses were performed with SPSS software (version 22, IBM Corporation, Armonk, NY, USA).

\section{Results}

The neurological and neuropsychological investigations provided results for only 65 of 77 recruited participants [Figure 1]. Twelve individuals withdrew from further

Table 1: Descriptive statistics of outcome measures from Cambridge Neuropsychological Test Automated Battery subtests

\begin{tabular}{lcc}
\hline CANTAB subtests & \multicolumn{2}{c}{ Statistics } \\
& Mean & SD \\
\hline IED1 total errors & 14.66 & 10.445 \\
IED2 total trials & 76.48 & 17.618 \\
RVP1 total misses & 11.48 & 4.373 \\
RVP2 total hits & 15.77 & 3.927 \\
RVP3 mean latency & 524.45 & 110.001 \\
SOC1 problems solved in minimal number of moves & 7.71 & 1.860 \\
SOC2 initial thinking time for 4-moves problems & $11,559.51$ & $12,133.018$ \\
SOC3 initial thinking time for 5-moves problems & $12,636.95$ & 9639.114 \\
SOC4 subsequent thinking time for 4-moves problems & 5411.43 & 6038.130 \\
SOC5 subsequent thinking time for 5-moves problems & 4515.26 & 9366.353 \\
SWM1 total errors & 41.65 & 19.615 \\
SWM2 strategy & 35.86 & 5.006
\end{tabular}

SD - Standard deviation, SWM - Spatial Working Memory, SOC - Stockings of Cambridge, RVP - Rapid Visual Information Processing, IED - Intra-Extra Dimensional set, CANTAB - Cambridge Neuropsychological Test Automated Battery participation in the study before the neuropsychological investigation and were excluded from the analyses. The main results are summarized in Tables 1-3.

The values of 12 outcome measures for the four CANTAB battery subtests were included in the analyses [Table 1]. The relationships between the different outcome measures (correlation coefficients) are presented in Table 2. Very high correlation was found between the two outcome measures for IED showing executive impairments - total errors and total trials $(R=0.948, P<0.05)$. There was also a high correlation $(R=0.698, P<0.05)$ between the two outcome measures SWM1 and SWM2 (i.e. total errors and strategy). Such correlations were indicative of internal consistency of the testing results.

According to the CANTAB results received, we also studied the distributions of subjects by the number of outcome measures with results below the norms. The distribution was not normal $(P<0.01)$ [Figure 2].

The computer system CANTAB-Eclipse had been validated and found sensitive to detect mild cognitive impairments. ${ }^{[4]}$ The participants in our study have been divided into two groups according to the results for "presence" or "absence" of MDCI. One of the subgroups included the subjects who performed well, having only up to two measures below the age-standardized normative data $(n=35,53.8 \%)$. The participants in the other subgroup $(n=30,46.2 \%)$ had 3 or more outcome measures below the normative data, i.e., performance being worse than the standard population results and therefore, were considered to have a MDCI. The median of the whole sample was two measures below the norms.

Further, more detailed percentage distribution of the participants was the following: 9 (13.8\%) subjects showed

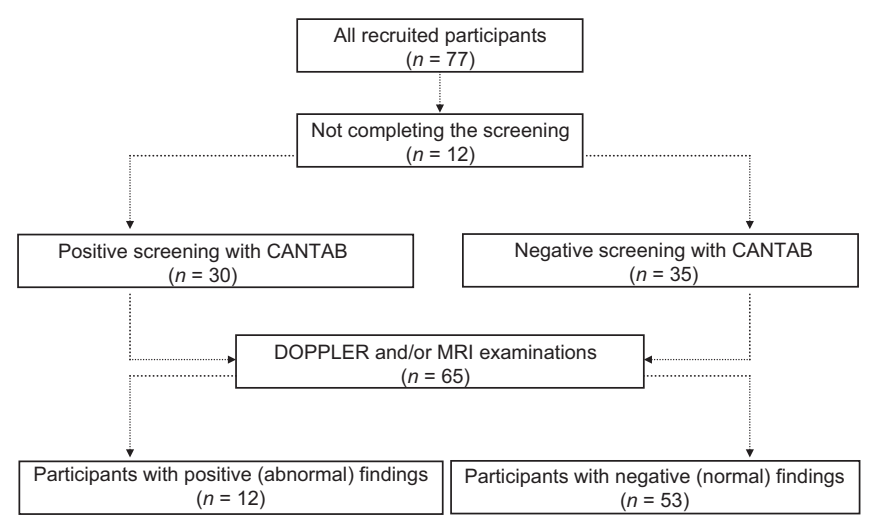

Figure 1: CONSORT flowchart of study design. MRI - Magnetic resonance imaging, Doppler ultrasound, SCVD - Subclinical cerebrovascular disease 
Atanassova, et al.: Early cognitive impairments in cerebrovascular disease

Table 2: Correlation between the results of the various subtests of the Cambridge Neuropsychological Test Automated Battery

\begin{tabular}{|c|c|c|c|c|c|c|c|c|c|c|c|c|}
\hline Subtests & IED1 & IED2 & RVP1 & RVP2 & RVP3 & SOC1 & SOC2 & SOC3 & SOC4 & SOC5 & SWM1 & SWM2 \\
\hline IED1 & - & $0.948 * *$ & -0.004 & 0.052 & 0.094 & -0.102 & -0.042 & -0.125 & -0.172 & -0.109 & 0.085 & 0.030 \\
\hline IED2 & $0.948^{* *}$ & - & -0.023 & -0.074 & 0.120 & -0.129 & -0.009 & -0.078 & -0.091 & -0.054 & 0.086 & -0.031 \\
\hline RVP1 & -0.004 & -0.023 & - & $-10.00^{* *}$ & $0.486^{*}$ & $-0.498^{* *}$ & $-0.284^{*}$ & $-0.253^{*}$ & -0.150 & 0.206 & 0.220 & 0.154 \\
\hline RVP2 & 0.052 & -0.074 & $-10.00^{* *}$ & - & $-0.486^{*}$ & $0.484^{* *}$ & 0.249 & 0.223 & 0.145 & -0.193 & -0.197 & -0.123 \\
\hline RVP3 & 0.094 & 0.120 & $0.486^{* *}$ & $-0.486^{* *}$ & - & -0.241 & -0.220 & -0.163 & 0.003 & 0.112 & 0.032 & 0.014 \\
\hline SOC1 & -0.102 & -0.129 & $-0.498^{* *}$ & $0.484^{* *}$ & -0.241 & - & 0.193 & 0.168 & -0.226 & $-0.385^{* *}$ & -0.304 & $-0.266^{*}$ \\
\hline SOC2 & -0.042 & -0.009 & $-0.284^{*}$ & 0.249 & -0.220 & 0.193 & - & $0.490^{* *}$ & $0.467^{* *}$ & -0.042 & -0.014 & -0.176 \\
\hline SOC3 & -0.125 & -0.078 & $-0.253^{*}$ & 0.223 & -0.163 & 0.168 & $0.490^{* *}$ & - & $0.383^{* *}$ & 0.061 & -0.123 & -0.209 \\
\hline SOC4 & -0.172 & -0.091 & -0.150 & 0.145 & 0.003 & -0.226 & $0.467^{* *}$ & $0.383^{* *}$ & - & 0.244 & $0.265^{*}$ & 0.175 \\
\hline SOC5 & -0.109 & -0.054 & 0.206 & -0.193 & -0.112 & $-0.385^{* *}$ & -0.042 & 0.061 & 0.244 & - & $0.273^{*}$ & 0.175 \\
\hline SWM1 & 0.085 & 0.086 & 0.220 & -0.197 & 0.032 & $-0.304^{*}$ & -0.014 & -0.123 & $0.265^{*}$ & $0.273^{*}$ & - & $0.698^{* *}$ \\
\hline SWM2 & 0.030 & -0.031 & 0.154 & -0.123 & 0.014 & $-0.266^{*}$ & -0.176 & -0.209 & 0.175 & 0.175 & $0.698^{* *}$ & - \\
\hline
\end{tabular}

${ }^{*} P<0.05,{ }^{* *} P<0.01$. IED1 - Total errors, IED2 - Total trials, RVP1 - Total misses, RVP2 - Total hits, RVP3 - Mean latency, SOC1 - Problems solved in minimum moves, SOC2 - Initial thinking time-4 moves, SOC3 - Initial thinking time-5 moves, SOC4 - Subsequent thinking time-4 moves, SOC5 - Subsequent thinking time-5 moves, SWM1 - Total errors, SWM2 - Strategy, SWM - Spatial working memory, SOC - Stockings of Cambridge, RVP - Rapid visual information processing, IED - Intra-extra dimensional set

Table 3: Main characteristics of Cambridge Neuropsychological Test Automated Battery patients according to outcomes, obtained by Doppler and magnetic resonance imaging investigations

\begin{tabular}{lccc}
\hline Variable (unit)* & Positive Doppler and/or MRI & Negative Doppler and MRI & Total \\
\hline Number (\%) & $41(63.1)$ & $24(36.9)$ & $65(100)$ \\
Gender (\%) (male/female) & $11(26.8) / 30(73.2)$ & $4(16.7) / 20(83.3)$ & $15(23.1) / 50(76.9)$ \\
Age (years) & $60.0 \pm 5.9$ & $56.3 \pm 5.2$ & $58.7 \pm 5.9$ \\
CVRF (\%) (yes/no) & $33(80.5) / 8(19.5)$ & $9(37.5) / 15(62.5)$ & $42(64.6) / 23(35.4)$ \\
Nonspecific symptoms (\%) (yes/no) & $35(85.4) / 6(14.6)$ & $9(37.5) / 15(62.5)$ & $44(67.7) / 21(32.3)$ \\
CVRF or nonspecific symptoms or both (\%) (yes/no) & $41(100.0) / 0(0.0)$ & $18(75.0) / 6(25.0)$ & $59(90.8) / 6(9.2)$ \\
MRI (\%) (yes/no)** & $18(66.7) / 9(33.3)$ & $0(0.0) / 7(100.0)$ & $18(52.9) / 16(47.1)$ \\
Doppler (\%) (yes/no) & $38(92.7) / 3(7.3)$ & $0(0.0) / 24(100.0)$ & $38(58.5) / 27(41.5)$ \\
Arterial hypertension (\%) (moderate/mild/no) & $15(36.6) / 18(43.9) / 8(19.5)$ & $0(0.0) / 4(16.7) / 20(83.3)$ & $15(23.1) / 22(33.8) / 28(43.1)$ \\
CVD (\%) (yes/no) & $22(53.7) / 19(46.3)$ & $4(16.7) / 20(83.3)$ & $26(40.0) / 39(60.0)$ \\
Diabetes mellitus (\%) (yes/no) & $15(36.6) / 26(63.4)$ & $2(8.3) / 22(91.7)$ & $17(26.2) / 48(73.8)$ \\
Dyslipidemia (\%) (yes/no) & $3(12.5) / 21(87.5)$ & $16(39.0) / 25(61.0)$ & $19(29.2) / 46(70.8)$ \\
Family history (\%) (yes/no) & $14(34.1) / 27(65.9)$ & $2(8.7) / 21(91.3)$ & $16(25.0) / 48(75.0)$ \\
CANTAB (\%) (yes/no) & 18 (50.) & $12(50.0) / 12(50.0)$ & $30(46.2) / 35(53.8)$ \\
\hline
\end{tabular}

${ }^{*}$ Number or frequency (percentage) or mean $\pm S D$, as appropriate; The category "yes" refers to presence of a symptom, pattern or positive (pathological) result while the category "no" refers to absence of the symptom, pattern or a negative (normal) result, **Data missing for MRI in 31 patients, ***The CANTAB category "yes" refers to 3-7 outcome measures with results below the norms and implies the presence of cognitive impairments, while the category "no" refers to 0-2 outcome measures with results below the norms only and implies absence of cognitive impairments, \#Based on CANTAB test results and the presence of above characteristics (CVRF, nonspecific symptoms, Doppler, MRI), suspected cases with SCVD have been identified with the following distribution: 12 SCVD (yes)=18.5\% and 53 SCVD (no)=81.5\% [Figure]. CANTAB - Cambridge Neuropsychological Test Automated Battery, CVRF - Cerebrovascular risk factor, MRI - Magnetic resonance imaging, SCVD - Subclinical cerebrovascular disease, CVD - Cerebrovascular disease

normal performance; $26(40.0 \%)$ of the subjects had up to two outcome measures below the age norms; while 30 subjects $(46.2 \%)$ had from 3 (10 subjects, $15.4 \%)$ to 7 (1 subject, 1.5\%) measures below the norms.

\section{Clinical characteristics and risk factors}

The mean age of participants was 58.7 (SD 5.9) years which may be considered at risk for $\mathrm{MDCl}$ and possible hidden positive findings on Doppler/MRI. The risk factor questionnaire revealed nonspecific symptoms in 44 (67.7\%) of the subjects (a total of 139 symptoms). In 42 (64.6\%) subjects, we found at least one of the conventional CVRFs (arterial hypertension, ischemic heart disease, diabetes mellitus, dyslipidemia, family history for vascular accidents and dementia, etc.).
Abnormal lipid profile was found in 19 (29.2\%) of all subjects [Table 3] with the mean total cholesterol of $6.78(0.22) \mathrm{mmol} / \mathrm{L}$ against $5.15(0.68) \mathrm{mmol} / \mathrm{L}$ in the remaining subjects without dyslipidemia $(P<0.001)$. The level of triglycerides was also significantly higher $(2.14 \pm 0.73 \mathrm{mmol} / \mathrm{L})$ than that in the 46 subjects without dyslipidemia $(1.41 \pm 0.38)$.

Abnormal findings from the extra- and trans-cranial Doppler examination have been established in 38 (58.5\%) of all the studied subjects [Table 3]. Thirty-four subjects (52.3\%) agreed to be examined by brain MRI. In 12 (35.3\%) subjects, MRI revealed lacunar infarcts in the deep brain structures, in $2(5.9 \%)$ subjects, single larger asymptomatic ischemic lesions, and in $8(12.3 \%)$, initial cortical atrophy. 
In particular, more detailed statistical distribution analysis indicated that among all $44(67.7 \%)$ subjects with nonspecific symptoms, the most frequent complaint was intermittent attacking headache (28 out of 44 subjects, $63.6 \%$ ), sleep disturbances ( $n=26,59.1 \%)$, and sensation of "rapid mental fatigue" ( $n=25,56.8 \%)$. These three most frequent nonspecific symptoms were reported as related to mild and moderate arterial hypertension (in 37 of 65 subjects, 56.9\%). Among all the studied conventional CVRFs, mild and moderate arterial hypertension was the most frequent, as was ischemic heart disease detected by electrocardiogram ( $n=26,40 \%)$ as well as dyslipidemia detected by high total serum cholesterol and triglycerides $(n=19,29.2 \%)$.

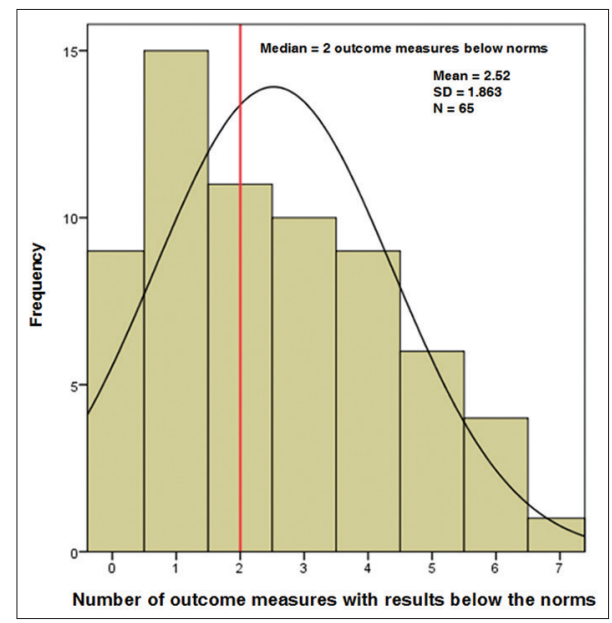

Figure 2: Histogram of the distribution of outcome measures with results below the norms. Red vertical line, median. SD - Standard deviation, $N$ - Number of study subjects
The prevalence of CVRFs and nonspecific symptoms was strongly related to the positive (abnormal) findings for SCVD from the Doppler/MRI examinations $(P<0.001)$, [Table 4]. In 38 (58.5\%) of all subjects who were evaluated by Doppler examination, we found mild to moderate asymptomatic stenosis of the extra-and trans-cranial brain vessels.

\section{Comparative analysis}

According to the above positive (abnormal) instrumental findings, the participants with either positive Doppler examination or MRI, or both, were classified into two outcome groups: With positive findings $(n=41,63.1 \%)$ and with negative findings $(n=24,36.9 \%)$ [Table 3]. The distribution of each of the suspected predictive factors according to the outcome was presented in a comparative and univariate analysis as shown in Table 4, thus giving the basis for cross-tabulation and identification of the subjects who had more than one risk factor or pathological (abnormal) finding. After the neuropsychological screening, the 30 (46.2\%) subjects with outcome measures below the norms were considered with MDCIs, i.e. abnormal (positive, "yes" CANTAB). According to the cross-tabulations, $12(40 \%)$ of the 30 subjects with MDCls also had positive outcome from Doppler examination and/or MRI as well as the presence of CVRFs and nonspecific symptoms (or, 18.5\% of all 65 subjects).

\section{Univariate and multivariate modelling}

The univariate analysis indicated which risk factors, symptoms or laboratory parameters were significantly associated with

Table 4: Univariate logistic regression analysis to predict Doppler and/or magnetic resonance imaging findings

\begin{tabular}{|c|c|c|c|}
\hline Characteristics/risk factors & Categories & $\begin{array}{l}\text { Prevalence of outcome within } \\
\text { the factor categories (\%) }\end{array}$ & OR $(95 \% \mathrm{CI}), P^{*}$ \\
\hline Gender & Male/female & $73.3 / 60.0$ & $0.545(0.152-1.955), 0.352$ \\
\hline $\operatorname{Age}^{*}$ & - & - & $1.130(1.020-1.251), 0.019$ \\
\hline CVRF $^{*}$ & Yes/no & $78.6 / 34.8$ & $6.875(2.218-21.307), 0.001$ \\
\hline Nonspecific symptoms* & Yes/no & $79.5 / 28.6$ & $9.722(2.937-32.184),<0.001$ \\
\hline Arterial hypertension* & Moderate/mild/no & $100 / 81.8 / 28.6$ & $13.39(3.89-46.09),<0.001$ \\
\hline Heart disease ${ }^{*}$ & Yes/no & $84.6 / 48.7$ & $5.789(1.681-19.93), 0.005$ \\
\hline Diabetes mellitus* & Yes/no & $88.2 / 54.2$ & $6.346(1.306-30.83), 0.022$ \\
\hline Dyslipidemia* & Yes/no & $84.2 / 54.3$ & 4.480 (1.147-17.503), 0.031 \\
\hline Family history* & Yes/no & $87.5 / 56.3$ & $5.444(1.113-26.633), 0.036$ \\
\hline CANTAB (outcome measures with 0-7 results below the norms) & - & - & $1.011(0.769-1.328), 0.939$ \\
\hline CANTAB (outcome measures with 0-7 results below the norms) & Yes $(3-7) /$ no $(0-2)$ & $60.0 / 65.7$ & $0.783(0.285-2.149),<0.634$ \\
\hline SWM1 total errors* & - & - & $1.028(1.000-1.057), 0.048$ \\
\hline IED1 total errors (adjusted) & - & - & $1.029(0.985-1.075), 0.193$ \\
\hline IED2 total trials (adjusted) & - & - & $1.015(0.992-1.040), 0.205$ \\
\hline IED total ratio (errors/trials) & - & - & $1.065(0.989-1.146), 0.097$ \\
\hline IED total ratio* SWM1 total errors ${ }^{*}, * *$ & - & - & $1.002(1.000-1.003), 0.012$ \\
\hline IED total ratio* SWM1 total errors (binary) & Yes $/ \mathrm{no}^{* * *}$ & $81.6 / 37.0$ & $7.529(2.426-23.37),<0.001$ \\
\hline
\end{tabular}

${ }^{*} \mathrm{OR}$ with $95 \% \mathrm{Cl}$ at $P<0.05,{ }^{*}$ Combined predictor, ${ }^{* *}$ Yes, category with values above the best cut-off of 508.8 ; Number category with values at or below the best cut-off of 508.8. CVRF - Cerebrovascular risk factors, OR - Odds ratio, Cl - Confidence interval, CANTAB - Cambridge Neuropsychological Test Automated Battery, SWM - Spatial working memory, IED - Intra-extra dimensional set 
the positive outcome findings, i.e. abnormal results from Doppler examination and/or MRI [Table 4]. Understandably, the strongest potential predictors were arterial hypertension $(O R=13.4)$, nonspecific symptoms $(O R=9.72)$, and presence/absence of CVRFs (OR $=6.88)$.

Only two of the subtests of CANTAB tools appeared to be potentially promising (although weak) predictors of the outcome as found at the univariate analysis (SWM1 total errors; IED1 total errors [adjusted]; IED2 total trials [adjusted]). At the multivariate analysis, these potential, individual variables appeared to be independent predictors [Table 5] and allowed the exploration of second and further level interactions and possibilities to create combined predictors. In this way, at the first stage, we derived a ratio of the adjusted total trials and errors (IED1/IED2) and found that its relationship, as a derived predictor of the outcome, was of marginal significance [Table 4] (IED total ratio [error/trials], $P=0.097$ ).

Furthermore, to improve predictivity of the models with the participation of the CANTAB tool subtests, we studied various combinations, of different levels, between the independent predictors. Finally, we established that the best accuracy of $82.5 \%$ was achieved by a multifactor interaction logistic regression model, with CVRF and combined CANTAB predictor "IED total ratio [errors/trials] $\times$ SWM1 total errors" [Table 5] (3D-type, $P=0.006$ ).

As a single predictor, CNPP had also a statistically significant predictive value [Figure 3 and Table 4] $(P=0.012)$. The fitting line of logistic equation was used to illustrate the relationship [Figure 3a] where 1 unit increase in CNPP was associated with $0.20 \%$ increase in the probability of the outcome. To further confirm its predictive value, we performed a receiver operating characteristic (ROC) curve analysis (area under the ROC curve $\left[\mathrm{AUC}_{\mathrm{ROC}}\right]=0.713$, 95\% $\mathrm{Cl}$ 0.58-0.85) and established its best cutoff value as a combined predictor at 508.8 units [Figure 3b]. This was very close to the intersection of the red logistic regression line and the black horizontal probability line of 0.5 , above which the outcome is usually classified as positive (abnormal) according to the Doppler/MRI examinations [blue vertical line at 508.8 units on Figure 3a]. We then went further to establish the classification (predictive) feasibility of the combined prognostic factor and created a binary predictor with two risk levels according to its best cut-off value ("no" = absence or low-risk <508.8; and "yes" = presence or high-risk $\geq 508.8$ units). As a binary predictor (yes/no), the relationship to and predictivity for CNPP of the outcome were clearly established $(\mathrm{OR}=7.53,95 \% \mathrm{CI} 2.42-23.4$, $P<0.001$ ) [Table 4].

\section{Discussion}

The knowledge about SCVD could help the general clinical practice, the prognosis and prevention of vascular cognitive impairment, and further, stroke and dementia. Prevention is related to the possibility of identifying the individuals at increased risk.

Vascular cognitive impairment is a clinical diagnosis but there are no uniformly accepted clinical criteria for it. ${ }^{[6]}$ The $\mathrm{MDCl}$ of vascular type is not recognizable during the routine clinical neurological examination; it has been accepted as the earliest symptom of SCVD and can be revealed by specific neuropsychological testing. ${ }^{[7]}$ The most frequently impaired functions in vascular MDCls are the attention, delayed recall, visual-constructive praxis, and executive functions. ${ }^{[8,9]}$ During the recent years, to detect more precisely, and with greater specificity, such early signs and symptoms of cognitive impairments, a number of computer-based self-assessment neuropsychological tests have been developed. Their advantages are based on the limited communication of the investigator with the patient, steeper learning curve of the tested subjects and faster calculation and analysis of the results..$^{[10]}$

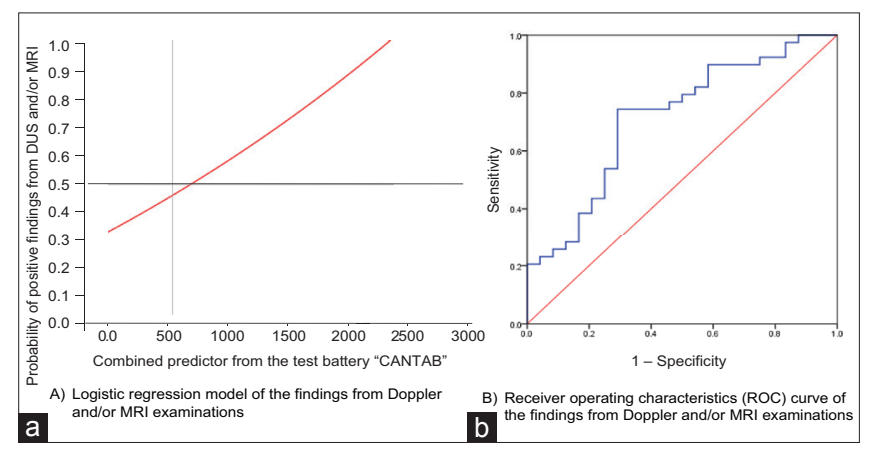

Figure 3: Models of Doppler and/or magnetic resonance imaging findings and the combined neuro-psychological factor in healthy adults. (a) Probability of positive Doppler ultrasound and magnetic resonance imaging findings expressed as a nonlinear relationship along the combined neuropsychological factor range $\left(F_{\text {model }}=7.49, P_{\text {model }}=0.08\right)$. X-axis, combined neuropsychological factor; $Y$-axis, probability of positive findings (where 0.0 = no positive finding; 1.0 = positive finding); horizontal line, cut-off event probability of 0.5 . (b) Receiver operating characteristic curve of the Doppler/magnetic resonance imaging findings versus combined neuropsychological factor: For a given combined neuropsychological factor level, the ordinate values indicate the corresponding true-positive rate (fraction of subjects with positive findings with this combined neuropsychological factor) and the abscissa values indicate the corresponding part of the false-positive rate (fraction of subjects without positive findings with this combined neuropsychological factor). The inflection point of the curve was chosen as the optimal diagnostic value. The larger area between the receiver operating characteristic curve and the diagonal line reflects the higher degree with which the combined neuropsychological factor parameter shows a predictive benefit. X-axis, 1-specificity; $Y$-axis, sensitivity. Both estimates are expressed as a proportion of subjects without or with positive findings (i.e., from 0.00 to 1.00). MRI - Magnetic resonance imaging, DU - Doppler ultrasound 
Table 5: Three-level logistic backward regression analysis of Doppler and/or magnetic resonance imaging findings in 65 healthy subjects $^{\#}$

\begin{tabular}{|c|c|c|c|}
\hline Independent variable ${ }^{\$}$ & Accuracy (\%) & OR (95\% CI) & $P$ \\
\hline \multicolumn{4}{|l|}{$1^{\text {st-level models (ordinary) }}$} \\
\hline \multicolumn{4}{|l|}{ 1A-type (2 single predictors, CANTAB only) } \\
\hline *SD_10 & 73.4 & $1.589(0.984-2.567)$ & 0.058 \\
\hline SWM1 total errors & & $1.035(1.005-1.067)$ & 0.023 \\
\hline \multicolumn{4}{|l|}{ 1B-type (2 single predictors) } \\
\hline CVRF & 76.9 & $7.742(2.310-25.952)$ & 0.001 \\
\hline SWM1 total errors & & $1.033(1.001-1.066)$ & 0.046 \\
\hline \multicolumn{4}{|l|}{ 1C-type (2 single predictors) } \\
\hline CVRF & 79.4 & $9.281(2.629-32.771)$ & 0.001 \\
\hline IED1 total errors (adjusted) & & $1.042(0.995-1.092)$ & 0.083 \\
\hline \multicolumn{4}{|l|}{$2^{\text {nd }-l e v e l ~ m o d e l s ~(o r d i n a r y, ~ w i t h ~ r a t i o ~ o f ~ p r e d i c t o r s) ~}$} \\
\hline \multicolumn{4}{|l|}{ 2A-type (3 single predictors) } \\
\hline Nonspecific & 76.2 & $10.178(2.779-37.282)$ & $<0.001$ \\
\hline SWM1 total errors & & $1.024(0.992-1.056)$ & 0.143 \\
\hline IED total ratio (errors/trials) & & $1.053(0.971-1.141)$ & 0.214 \\
\hline \multicolumn{4}{|l|}{ 2B-type (2 single predictors) } \\
\hline CVRF & 79.4 & $10.035(2.77536 .282)$ & $<0.001$ \\
\hline IED total ratio (errors/trials) & & $1.090(1.0021 .185)$ & 0.044 \\
\hline \multicolumn{4}{|l|}{ 2C-type (3 single predictors) } \\
\hline CVRF & 81.0 & $10.576(2.713-41.227)$ & 0.001 \\
\hline IED total ratio (errors/trials) & & $1.077(0.992-1.170)$ & 0.077 \\
\hline SWM1 total errors & & $1.030(0.996-1.065)$ & 0.082 \\
\hline \multicolumn{4}{|l|}{$3^{\text {rd }}$-level models (with interactions) } \\
\hline \multicolumn{4}{|l|}{ 3A-type (single predictors and their interactions) } \\
\hline Nonspecific symptoms & 77.8 & $9.760(2.654-35.893)$ & 0.001 \\
\hline IED total ratio (errors/trials) & & $0.990(0.887-1.104)$ & 0.852 \\
\hline IED total ratio (errors/trials) $\times$ SWM1 total errors & & $1.002(1.000-1.004)$ & 0.110 \\
\hline \multicolumn{4}{|l|}{ 3B-type (single predictors and their interactions) } \\
\hline CVRF & 81.0 & $14.316(3.261-62.849)$ & $<0.001$ \\
\hline IED total ratio (errors/trials) & & $0.972(0.867-1.090)$ & 0.630 \\
\hline IED total ratio (errors/trials) $\times$ SWM1 total errors & & $1.003(1.000-1.005)$ & 0.019 \\
\hline \multicolumn{4}{|l|}{ 3C-type (interactions only) } \\
\hline Nonspecific symptoms & 76.2 & $9.790(2.663-35.994)$ & 0.001 \\
\hline IED total ratio (errors/trials) $\times$ SWM total errors & & $1.002(1.000-1.003)$ & 0.049 \\
\hline \multicolumn{4}{|l|}{ 3D-type (interactions only) } \\
\hline CVRF & 82.5 & $13.988(3.220-60.759)$ & $<0.001$ \\
\hline IED total ratio (errors/trials) $\times$ SWM1 total errors & & $1.002(1.001-1.004)$ & 0.006 \\
\hline
\end{tabular}

"Doppler and/or MRI findings ( $n=41$ cases) is the outcome ordinal variable with dichotomous coding (positive=1, negative $=0$ ) where "positive" refers to "pathological" and "negative" refers to "normal" results, "Only the variables that were significant at $P<0.05$ from the univariate analyses, had marginal probabilities and/or considered clinically important were included as potentially independent predictors in the initial models. The best final $3^{\text {rd }}$ level (interaction) models with 2 predictors $(3$ variables, type $3 \mathrm{C}$ and type $3 \mathrm{D}$, respectively) were derived where the type 3D had the highest statistical significance and accuracy of $82.5 \%$ (see text for more explanations). CVRF - Cerebrovascular risk factors, OR - Odds ratio, $\mathrm{Cl}$ - Confidence interval, CANTAB - Cambridge Neuropsychological Test Automated Battery, SWM - Spatial working memory, IED - Intra-extra dimensional set, MRI - Magnetic resonance imaging, ${ }^{*}$ SD_10 - Standard deviation_10 is the standard deviation of SOC subsequent thinking time 5 moves (one of the SOC subtests).

Following Diagnostic and Statistical Manual of Mental Disorders-V criteria (2013), a prominent decline in complex attention and executive functions is expected in mild vascular neurocognitive disorder, the profile of the cognitive impairment in this type of disorders being different from the cognitive profile in neurodegenerative disorders. ${ }^{[11-14]}$ In scientific literature, deficit in the verbal episodic memory and dysexecutive syndrome are the most expressed mild cognitive impairments of vascular origin. ${ }^{[15]}$ The vascular MDCI, with a specific profile, as the "earliest symptom" of SCVD has to be one of the priorities of the researchers..$^{16,17]}$

One of the most recent neuroepidemiological studies of dementias in Bulgaria established that the prevalence of vascular cognitive impairment (26\%) is higher than that in most European countries. ${ }^{[18,26]}$ Other epidemiological study also confirmed that the widely known CVRFs - arterial hypertension, diabetes mellitus, obesity, smoking, 
dyslipidemia and lack of physical activity - are prognostic factors not only for cerebrovascular events but also for vascular cognitive impairment and vascular dementia, ${ }^{, 19]}$ while vascular risk factors had much smaller effect in Alzheimer's disease than the genetic mechanisms of neurodegeneration. ${ }^{[20,21]}$

In the view of this relatively high stroke prevalence in Bulgaria as well as the increased prevalence of CVRFs among the middle-aged Bulgarian population, ${ }^{[22,23]}$ we have chosen the age interval from 51 to 65 years as the main inclusion criterion in our study. This age range has been assumed as "the age at risk"-a nonmodifiable CVRF which may have influenced or provoked the existence of early MDCI of vascular type. Wiederkehr et al., ${ }^{\mid 24]}$ found that nondemented elderly individuals aged more than 65 years, with 3 or more vascular risk factors, had bigger impairments of executive functions, abstract thinking and speed of information processing than elderly subjects without any risk factors.

Our findings related to nonspecific symptoms, most importantly CVRFs and Doppler examination, among all participants, as well as the MRI results, among more than a half of them, are in accordance with the data reported by earlier studies of Bulgarian and foreign authors for this part of the middle-aged population. ${ }^{[22,25-28]}$ According to CANTAB outcome measures with results below the age norm, almost half of all the studied subjects (30 participants, $46.2 \%$ ) were considered to have $\mathrm{MDCl}$, i.e., with $\geq 3$ outcome measures. They showed performance worse than the mean of their peer group. Twelve (18.5\%) of all 65 participants were found to have MDCIs, nonspecific symptoms, CVRFs, and positive findings at Doppler and/or MRI thus being considered at risk of vascular cognitive impairment and/or further strokes. ${ }^{[14,28]}$

We consider that two other sub-groups might be also indicated for prevention. One consisted of 12 (18.5\%) subjects with MDCI but without positive (abnormal) MRI/Doppler findings, while another group consisted of $6(9.2 \%)$ subjects with $\mathrm{MDCl}$ who had only CVRFs or nonspecific symptoms. We think that in these additional 18 subjects with MDCI (27.7\%) there is an increased probability of finding possible vascular cognitive impairment or cognitive impairment of the degenerative type.

Ten (15.4\%) subjects did not have MDCI but had MRI findings consistent with the SCVD profile. Although Moorhouse and Rockwood did not find a correlation between MDCIs and the existing asymptomatic ischemic brain lesions, ${ }^{[29]}$ we think these subjects should be considered a high-risk group and should also be closely monitored for prevention. Cerebral small-vessel disease is the most frequent silent (subclinical)
CVD. Vascular cognitive impairment correlates to a great extent, with small-vessel disease, which can be visualized on MRI studies as lacunar infarcts, white matter lesions, cerebral microbleeds and cortical atrophy. ${ }^{[30]}$ In more than 2/3 of our patients, MRI findings were consistent with subcortical CVD, 14 (41.2\%) of them had silent brain infarcts, and 30 subjects $(46.2 \%)$ had MDCls. Another study reported that half of the patients with a first-ever lacunar infarct have mild cognitive impairment of subcortical vascular features and its presence may be a predictor of subcortical vascular dementia in the medium-long-term. ${ }^{[31]}$

Some of the investigations in this field have underlined the importance of the complex neurological, neurosonographic, and MRI examinations of the brain to confirm the profile of $\mathrm{MDCl}$ in adult individuals. Fromm et al. ${ }^{[16]}$ have studied a small number of patients $(n=10)$ with a history of MDCI. CVRFs have been found in 6 of the persons, and positive Doppler findings for atherosclerotic lesions - in 4 persons (in 3 - light, and in 1 - moderate carotid stenosis). The MRI established small chronic ischemic lesions in 1 person, cortical atrophy in 4 persons, smaller volume of the hippocampus in 1 person and multiple subcortical infarcts in 3 persons. It should be noted that CVRFs are also present in patients with Alzheimer's disease; therefore, it is very difficult to distinguish the MDCI of vascular type from those of degenerative type in clinically healthy persons.

In this sense, the validation of our newly-derived CNPP and its practical application would facilitate the identification and closer monitoring of individuals at increased risk. Its use may also allow differentiating more correctly, the patients with newly-revealed MDCIs of vascular type. The features of MDCIs, we found, correspond to the parameters described by Sikaroodi et al. ${ }^{[8]}$ In their study, the mean values of Montreal Cognitive Assessment score were significantly lower in patients with two or more CVRFs compared with those with only one risk factor, with delayed recall and visual-executive functions being most frequently impaired. The most frequent impairment from the Mini Mental State Examination test was attention.

Based on the above described reasoning, our study analyzed 12 outcome measures from 4 sub-tests of the CANTAB battery and we established that a relatively high percentage of the screened individuals had MDCIs $(n=30,46.2 \%)$. We also tested further the predictive role of each of these variables in view of the positive (abnormal) findings from Doppler/ MRI, but it was found not convincing. Only one measure of the CANTAB subtests (SWM total errors) was significantly, although weakly, associated with the positive Doppler/MRI findings $(\mathrm{OR}=1.028, P=0.048)$. This finding directed our 
attention to possible correlations between the outcome measures, showing the performance on the CANTAB subtests. We established moderate to strong correlations between the variables measuring the attentional set shifting in solving executive tasks and in rule acquisition (IED1 and IED2) and the measures of the working memory and strategy use (SWM1 and SWM2). Therefore, in a multivariate (multifactor) statistical analysis, we were able to establish the role of two of them (IED ratio total errors/trials, and SWM total errors), separately or in combination, in predicting the positive (abnormal) finding from Doppler/MRI, independently of each other.

In this way, we were able to create a new CNPP as based on the IED ratio and the SWM1 measure - the later combination may be easily explained as a natural multiplication of the effect of the working memory and executive functions in the process of finding solutions of the tasks. It also takes into account the time during which an abnormal number of errors are made. On this basis, we built a statistically significant prognostic model to predict findings from Doppler/MRI that are pathognomonic for SCVD. The model included the CNPP as well as the presence/absence of CVRFs that taken together, were in support of our hypothesis that completion of the CANTAB tests, measuring the memory, attention and executive functions, can predict existing subclinical Doppler/ MRI findings.

It should be noted that this newly created best predictive model, including CNPP and CVRF, did show an accuracy of $82.5 \%$ in the view of the positive (abnormal) findings from Doppler/MRI. The association of the CNPP (as part of the CANTAB tool) with the ultrasound/neuroimaging results is in support of the working hypothesis that the results from the CANTAB tests measuring the memory, attention and executive functions may reflect existing silent cerebrovascular damages of the brain. Of note, the components of the combined predictors coincide to a large extent with the description of Sikaroodi et al. ${ }^{[8]}$ which included disturbed distribution of attention, dysexecutive syndrome (elaboration of strategies, decision-making with suppression of inadequate ones), and impaired assessment of the feedback about the quality and sequence of the executed operations. The range of curve analyses revealed the best cutoff value of CNPP (508.8) according to which the testing of cognitive functioning may allow one to discriminate between the presence (high risk at $\geq 508$.8) or absence (low-risk at $<508.8$ ) of positive (abnormal) findings from Doppler/MRI examinations.

We consider the relatively limited sample studied as a limitation of our project. This pilot model, however, could be further tested in a prospective study with a larger, independent cohort. If further validated, our findings may be also explored as the basis of a new clinical prediction rule $^{|32|}$ to help physicians in neurological practice and primary care, better identify healthy adults with an increased risk for cognitive impairment, SCVD and early dementia, similar to the application of the $\mathrm{ABCD}^{2}$ rule after a TIA. ${ }^{[33]}$

\section{Conclusions}

We conclude that the present study with its neurological, neuropsychological, neurosonographic, and neuroimaging methods was able to identify cognitive impairments in half of the included clinically healthy, adult individuals. In about one-fifth of all the studied individuals, these cognitive impairments were associated with the existing CVRF, nonspecific symptoms, and abnormal findings on Doppler and/or MRI examinations.

Methodologically, our study has contributed to the evaluation of a range of factors for prediction of vascular damage to the brain. We identified a new CNPP based on the results from neuropsychological testing in adults at age at risk for SCVD. The prognostic model, including the combined predictor and the presence of CVRF, has shown a relatively high accuracy of $82.6 \%$ and may be used as a basis of a new clinical prediction rule to correctly identify persons that are at increased risk for SCVD and could be targeted for further neuroimaging examinations and closer monitoring. We should emphasize that the identification of subjects at increased risk for CVD and cognitive problems is of highest priority to better design and implement the necessary preventive measures at an individual level.

Our results confirmed the hypothesis that it is possible to identify, by noninvasive methods, the individuals at age at risk who have cognitive impairments, and to establish the significant relationship of these impairments with existing CVRFs, nonspecific symptoms, and subclinical abnormal brain Doppler/MRI findings. This allowed us to create a CNPP (best cut-off of 508.8), able to distinguish between the presence and absence of abnormal Doppler/MRI findings. The pilot prognostic model has a relatively high accuracy of $82.6 \%$ to predict the Doppler/MRI findings that may serve as biomarkers for SCVD subjects at age at risk (51-65 years).

\section{Financial support and sponsorship}

This study was supported by the University Grant Project NO-2/2004, Medical University, Plovdiv, Bulgaria.

\section{Conflicts of interest}

There are no conflicts of interest. 


\section{References}

1. Waldstein SR, Wendell CR, Lefkowitz DM, Siegel EL, Rosenberger WF, Spencer RJ, et al. Interactive relations of blood pressure and age to subclinical cerebrovascular disease. J Hypertens 2012;30:2352-6.

2. Sutton-Tyrrell K, Kuller LH, Matthews KA, Holubkov R, Patel A, Edmundowicz D, et al. Subclinical atherosclerosis in multiple vascular beds: An index of atherosclerotic burden evaluated in postmenopausal women. Atherosclerosis 2002;160:407-16.

3. Kuller LH, Meilahn E, Bunker C, Yong LC, Sutton-Tyrrell K, Matthews K. Development of risk factors for cardiovascular disease among women from adolescence to older ages. Am J Med Sci 1995;310 Suppl 1:S91-100.

4. Facal D, Rodríguez N, Juncos-Rabadán O, Manuel Caamaño J, Sueiro J. Use of the Cambridge neuropsychological test automated battery for the diagnosis of mild cognitive impairment. A pilot study in a Spanish sample. Rev Esp Geriatr Gerontol 2009;44:79-84.

5. Owen AM, Morris RG, Sahakian BJ, Polkey CE, Robbins TW. Double dissociations of memory and executive functions in working memory tasks following frontal lobe excisions, temporal lobe excisions or amygdalo-hippocampectomy in man. Brain 1996;119(Pt 5):1597-615.

6. Hua Z, Zhong Z. Diagnosis advances in vascular cognitive impairment. J Nanjing Med Univ 2009;23:224-7.

7. Di Legge S, Hachinski V. Vascular cognitive impairment (VCI). Progress towards knowledge and treatment. Dement Neuropsychol 2010;4:4-13.

8. Sikaroodi H, Yadegari S, Miri SR. Cognitive impairments in patients with cerebrovascular risk factors: A comparison of Mini Mental Status Exam and Montreal Cognitive Assessment. Clin Neurol Neurosurg 2013;115:1276-80.

9. Traikov L, Bodik S, Boler F, Smag A. Neuropsychological investigations in vascular cognitive impairments. Bulg Neurol 2005;5:303-6.

10. Roque DT, Teixeira RA, Zachi EC, Ventura DF. The use of the Cambridge neuropsychological test automated battery (CANTAB) in neuropsychological assessment: Application in Brazilian research with control children and adults with neurological disorders. Psychol Neurosci 2011;4:255-65.

11. American Psychiatric Association. Diagnostic and Statistical Manual of Mental Disorders. $5^{\text {th }}$ ed. Arlington, VA: American Psychiatric Association; 2013.

12. Traikov L, editor. Neuropsychological investigations in mild cognitive impairment. In: Early Diagnosis of Dementia in Degenerative Diseases. Sofia: Vulkan 4; 2012. p. 52-64.

13. Collie A, Maruff P. The neuropsychology of preclinical Alzheimer's disease and mild cognitive impairment. Neurosci Biobehav Rev 2000;24:365-74.

14. Petersen RC, Doody R, Kurz A, Mohs RC, Morris JC, Rabins PV, et al. Current concepts in mild cognitive impairment. Arch Neurol 2001;58:1985-92.

15. Black SE. Vascular cognitive impairment: Epidemiology, subtypes, diagnosis and management. J R Coll Physicians Edinb 2011;41:49-56.

16. Atanassova PA. Clinical, neuroepidemiological and prognostic aspects of minor ischaemic cerebrovascular accidents in patients at risk age. DMSc Thesis, Plovdiv; 2014.
17. Fromm A, Lundervold AJ, Moen G, Skulstad S, Thomassen L. A vascular approach to mild amnestic cognitive impairment: A pilot study. Acta Neurol Scand Suppl 2013;196:73-6.

18. Dimitrov I, Tzourio C, Milanov I, Deleva N, Traykov L. Prevalence of dementia and mild cognitive impairment in a Bulgarian urban population. Am J Alzheimers Dis Other Demen 2012;27:131-5.

19. Richard E, Moll van Charante EP, van Gool WA. Vascular risk factors as treatment target to prevent cognitive decline. J Alzheimers Dis 2012;32:733-40.

20. de Oliveira FF, Bertolucci PH, Chen ES, Smith MC. Assessment of risk factors for earlier onset of sporadic Alzheimer's disease dementia. Neurol India 2014;62:625-30.

21. DeCarli C. Clinically asymptomatic vascular brain injury: A potent cause of cognitive impairment among older individuals. J Alzheimers Dis 2013;33 Suppl 1:S417-26.

22. Hadjiev D. Mortality from Stroke. Forum Medicus; 21 January, 2014. Available from: http://www.forummedicus.com/archives/ all-publications/3714. [Last accessed on 2015 Mar 30].

23. Andonova SP. Asymptomatic ischaemic disturbances of cerebral circulation. Varna: IK "Gea Print"; 2011. p. 89-91.

24. Wiederkehr S, Laurin D, Simard M, Verreault R, Lindsay J. Vascular risk factors and cognitive functions in nondemented elderly individuals. J Geriatr Psychiatry Neurol 2009;22:196-206.

25. Atanassova P, Alexandrov A, Semerdjieva M. Cerebrovascular risk factors in healthy adults without cerebrovascular accidents. Neurorehabilitatzia 2008;2:72-5.

26. Fujishima M, Yao H, Terashi A, Tagawa K, Matsumoto M, Hara H, et al. Deep white matter lesions on MRI, and not silent brain infarcts are related to headache and dizziness of non-specific cause in non-stroke Japanese subjects. Intern Med 2000;39:727-31.

27. Gállego J, Martínez-Vila E. Asymptomatic cerebrovascular disease and systemic diagnosis in stroke, atherothrombosis as a disease of the vascular tree. Cerebrovasc Dis 2005;20 Suppl 2:1-10.

28. Thomas GN, Lin JW, Lam WW, Tomlinson B, Yeung V, Chan JC, et al. Increasing severity of cardiovascular risk factors with increasing middle cerebral artery stenotic involvement in type 2 diabetic Chinese patients with asymptomatic cerebrovascular disease. Diabetes Care 2004;27:1121-6.

29. Moorhouse P, Rockwood K. Vascular cognitive impairment: Current concepts and clinical developments. Lancet Neurol 2008;7:246-55.

30. Grau-Olivares M, Arboix A. Mild cognitive impairment in stroke patients with ischemic cerebral small-vessel disease: A forerunner of vascular dementia? Expert Rev Neurother 2009;9:1201-17.

31. Grau-Olivares M, Arboix A, Bartrés-Faz D, Junqué C. Neuropsychological abnormalities associated with lacunar infarction. J Neurol Sci 2007;257:160-5.

32. Keogh C, Wallace E, O'Brien KK, Galvin R, Smith SM, Lewis C, et al. Developing an international register of clinical prediction rules for use in primary care: A descriptive analysis. Ann Fam Med 2014;12:359-66.

33. Galvin R, Atanassova PA, Motterlini N, Fahey T, Dimitrov BD. Long-term risk of stroke after transient ischaemic attack: A hospital-based validation of the $\mathrm{ABCD}^{2}$ rule. BMC Res Notes 2014;7:281. 